\title{
Culture in vitro des blastodermes de Poulet - Rapports entre les feuillets embryonnaires et le milieu nutritif contenant de la gélose ${ }^{1}$
} par

\section{J. GALLERA}

Laboratoire d'Embryologie expérimentale, Institut d'Anatomie, Université de Genève

Avec 2 figures dans le texte.

Spratt (1947) préconise d'ajouter de l'agar, dissous dans la solution physiologique, au milieu de culture, pour lui conférer une certaine consistance. Les substances nutritives sont fournies par un mélange de vitellus et d'albumen. Le blastoderme détaché de la membrane vitelline est étalé sur ce substratum, sa face ventrale tournée vers le bas. Cette méthode de culture in vitro présente pourtant de sérieux désavantages; en particulier l'extension périphérique du blastoderme est toujours minime. En effet, comme NEw (1955) l'a démontré, elle est due à l'adhérence de la zone périphérique du feuillet externe (bord d'enveloppement) à la membrane vitelline. Ce bord s'élargit progressivement et, en glissant sur la membrane vitelline, étire littéralement le blastoderme tout entier. Nicolet et moi-même (1961), nous avons constaté que dans les conditions de culture de Spratt le rempart vitellin dépasse toujours un peu le bord du feuillet externe (ectophylle). Ce feuillet, détaché de la membrane vitelline, se contracte grâce à la forte adhésion entre ses éléments cellulaires, et il prend alors l'aspect d'un épithélium cubique sur toute sa surface, bien que normalement ses

1 Travail subventionné par le Fonds national suisse de la Recherche scientifique.

Rev. Suisse de Zool., T. 70, 1963. 
cellules périphériques soient fortement étirées et aplaties. Le feuillet interne (entophylle) est aussi anormal: de nombreuses cellules deviennent étoilées et pénètrent plus ou moins profondément dans l'agar imbibé de substances nutritives où elles forment un véritable réseau. Le rempart vitellin émet de grosses papilles dans le substratum, en particulier dans sa zone marginale. L'entophylle adhère si étroitement à la gélose que, si l'on cultive le blastoderme avec sa membrane vitelline sur le milieu de Spratt, l'entophylle fixé au substratum et étiré par le feuillet externe en extension, se déchire en de nombreux endroits.

Presque en même temps et indépendamment de nous, Spratt et HaAs (1960a) ont aussi constaté que l'adhérence de l'entophylle au substratum freine les mouvements morphogénétiques propres à ce feuillet et par conséquent dans leurs expériences récentes ils étalent les blastodermes en position inversée, la face dorsale contre le milieu. Ces expériences variées et nombreuses ont pour but l'analyse des mouvements morphogénétiques, en particulier dans le feuillet interne (Spratt et HaAs 1960) et l'explication causale de la polyembryonnie (Spratt et HaAs 1960 et 1962) résultant de la section des blastodermes très jeunes, prélevés sur des œufs non incubés. Les mouvements morphogénétiques ont été étudiés soit à l'aide de marques de charbon animal ou de carmin, soit, plus rarement, au moyen de marques colorées au bleu de Nil. Ces auteurs ont décelé de très vifs mouvements cellulaires dans l'entophylle en voie de formation; en revanche les déplacements du matériel formatif ont été de très petite envergure dans le feuillet externe durant la période de la constitution de la ligne primitive. Partant de ces observations, ils ont conclu que la ligne primitive ne correspond pas, ni du point de vue cinématique ni du point de vue fonctionnel, au blastopore, mais qu'elle représente un centre allongé de prolifération cellulaire. Autrement dit, Spratt et HaAs (1962) reviennent aux conceptions déjà anciennes de Wetzel (1929) qu'on croyait définitivement rejetées après la parution des études méthodiques et comparatives de Pasteels (1937).

La discussion de la théorie du développement embryonnaire proposée par Spratt et Haas dépasserait largement le cadre de cette note; je dois pourtant remarquer que ces auteurs se sont limités à examiner leur matériel presque exclusivement in toto. Quoique mis en éveil par le blocage qui survient dans l'entophylle lorsqu'il 
est en contact avec l'agar, ils n'ont pas essayé de savoir si un phénomène de ce genre pourrait influencer les mouvements morphogénétiques du feuillet superficiel. Ma note est précisément une réponse à ce problème, réponse qui aurait dû précéder l'interprétation des résultats, d'ailleurs fort instructifs qu'ils ont obtenus.

Huit blastodermes, prélevés sur des œufs (White Leghorn) non incubés et fraîchement pondus, sont cultivés in vitro exactement selon la méthode de Spratt et Haas. A titre de contrôle, 13 autres blastodermes sont sectionnés le long de leur axe céphalo-caudal présumé et les deux moitiés, repoussées à $3 \mathrm{~mm}$ environ l'une de l'autre, sont cultivées soit sur le milieu de Spratt et Haas soit selon le procédé de New employé couramment dans notre laboratoire. Rappelons que ce procédé consiste à cultiver le blastoderme, sa face dorsale en bas, sur la membrane vitelline légèrement tendue sur une couche d'albumen. Quoique le blanc d'œuf ne satisfasse pas les besoins nutritifs de l'embryon (lequel est pourtant capable de poursuivre son développement aux dépens de ses propres réserves), cette technique de culture in vitro est beaucoup plus proche des conditions normales de développement que celle de Spratt et Haas. En tout cas, l'extension périphérique du blastoderme est considérable et, comme nous le verrons, les moitiés de blastodermes cultivées dans ces conditions arrivent à former des embryons plus normaux que ceux obtenus sur le milieu contenant de l'agar. Tous les blastodermes, opérés ou non, sont dessinés après leur mise en culture et avant leur fixation. Les croquis sont exécutés à l'aide du tube de Wild dans des conditions standard et leur surface est mesurée au planimètre. Les blastodermes cultivés sur le milieu de Spratt et Haas sont fixés au Boin avec leur substratum et l'ensemble est étudié sur des coupes sériées colorées à l'hématoxyline d'Ehrlich-éosine.

En cultivant des moitiés de blastodermes sur la gélose, j'aj obtenu les mêmes résultats que Spratt et Haas. Les blastodermes opérés ont donné des couples d'embryon nains (3/10 à 4/10 de la longueur normale) dont les têtes convergent vers le plan de section. L'aire vasculaire n'est jamais complète puisqu'elle ne parvient pas à se former le long du bord cicatrisé.

L'expansion des hémiblastodermes cultivés sur la membrane vitelline est telle qu'ils fusionnent toujours. Dans l'ensemble ainsi reconstitué se développent deux embryons indépendants, chacun 
étant pourvu de sa propre aire vasculaire. Les corps embryonnaires sont en général bien développés et souvent atteignent les $7 / 10$ de la longueur normale. Comparons l'extension périphérique des blastodermes de ces deux séries à celles des embryons de même âge développés in ovo. Nous constatons qu'après 42 heures de culture

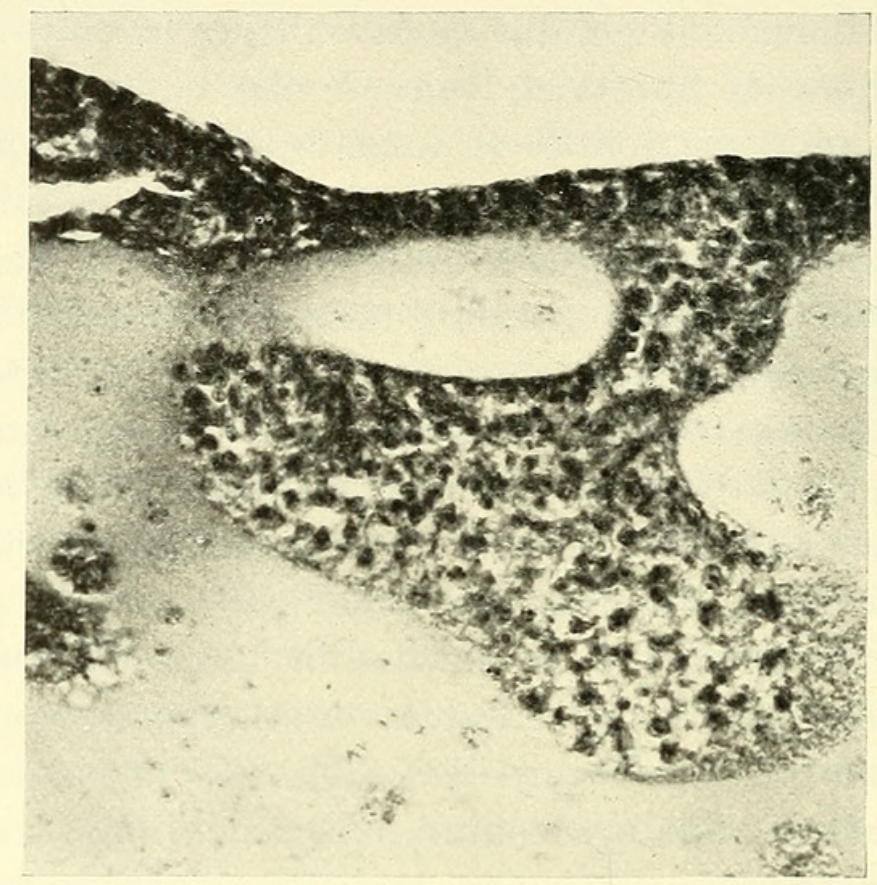

FIG. 1.

Coupe de la zone marginale d'un blastoderme fixé avec son substratum. Le rempart vitellin dépasse périphériquement le bord enroulé (à gauche sur la figure) de l'ectophylle. Sur la coupe on voit une grosse papille formée par les cellules du rempart.

sur la gélose, les blastodermes, aussi bien intacts que sectionnés, ont en moyenne à peine doublé de surface et n'atteignent qu'un douzième environ de la surface d'un blastoderme normal. En revanche, la surface des blastodermes cultivés selon la méthode de New a quintuplé pendant le même laps de temps, mais elle ne correspond qu'à la moitié de la surface d'un blastoderme de même âge incubé dans l'œuf.

L'analyse des coupes des blastodermes cultivés sur la membrane vitelline révèle que les feuillets embryonnaires, particulièrement le rempart vitellin, sont nettement amincis, mais, à part cette déficience imputable à la carence alimentaire, ils sont normalement constitués. 
Lorsque la face dorsale du blastoderme est adossée à l'agar, nous constatons après examen histologique que le rempart vitellin a franchi de toutes parts les limites de l'ectophylle. Une large frange d'entophylle se forme ainsi et elle émet une multitude de papilles s'enfonçant dans le milieu (fig. 1). Notons que nous avons déjà assisté à la formation de papilles similaires, quand l'entophylle était contre l'agar.

FIG. 2.

Coupe de l'ectophylle d'un blastoderme cultivé selon la méthode de Spratt et HaAs. La face dorsale de l'ectophylle est hérissée de fines micro-villosités, qui se sont détachées du substratum au moment de la fixation à la suite de la contraction du blastoderme.

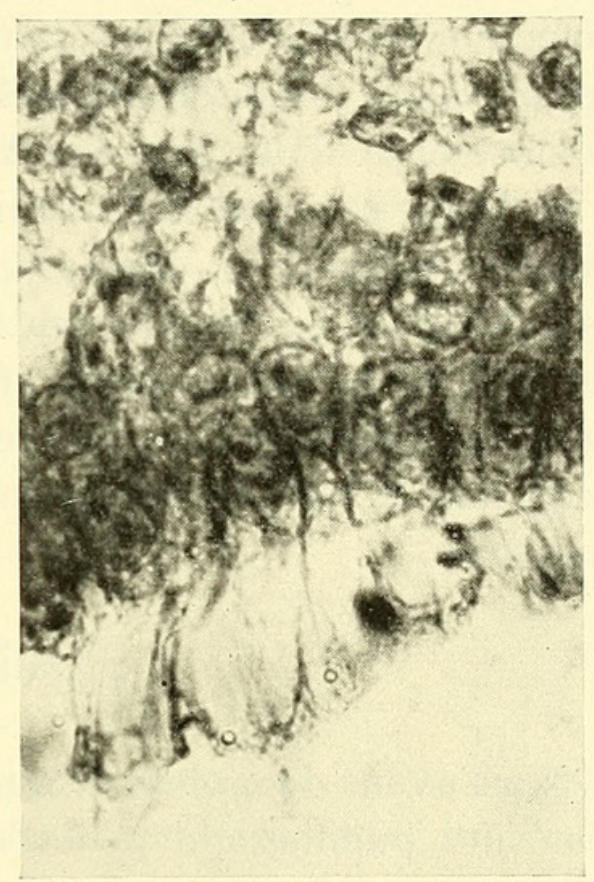

A considérer les surfaces, la légère extension enregistrée au cours du développement s'avère imputable au débordement périphérique du rempart vitellin. Donc l'ectophylle n'a, pour sa part, pratiquement pas changé de dimensions. Le bord de l'ectophylle s'enroule du côté du substratum (voir fig. 1 à gauche). L'épiblaste du corps embryonnaire est plus ou moins normalement différencié, tandis que partout ailleurs le feuillet externe est anormalement épais et prend la forme d'un épithélium cylindrique qui ne devient cubique que dans sa zone marginale. A l'exception de la zone embryonnaire, l'ectophylle se hérisse sur toute sa surface, tournée vers la gélose, de multiples et fines micro-villosités. Ces filaments, très réfringents et hyalins, adhèrent par leurs extrémités au substratum qui, à ce niveau, est plus coloré et granuleux. A la fixation, le blastoderme se contracte et le feuillet externe se détache du substratum en de 
nombreux endroits au niveau desquels ces micro-villosités, devenues des prolongements très étirés, sont particulièrement bien visibles (fig. 2). Dans d'autres régions, l'adhérence de l'ectophylle à l'agar est parfois telle que de larges lambeaux sont arrachés du blastoderme et restent fixés à l'agar.

Pour conclure, il m'apparaît que la forte adhérence de l'ectophylle à l'agar, l'absence d'extension de la part de ce feuillet et son épaisseur exagérée doivent inhiber considérablement les mouvements morphogénétiques dont l'ectophylle est le siège. Si le corps embryonnaire, minuscule toutefois, parvient à se constituer malgré ces obstacles, il le doit aux remarquables capacités régulatrices du matériel présomptif de la ligne primitive qui peut changer de destinée évolutive au cours de la formation de cette dernière.

A la suite de ces observations, reconnaissons qu'il nous faut accueillir avec une extrême réserve les conclusions surprenantes que Spratt et Haas tirent de leurs expériences effectuées dans des conditions si peu appropriées à l'étude des mouvements morphogénétiques.

\section{RÉSUMÉ}

Nous avons déjà observé que l'endoblaste du jeune blastoderme forme des papilles qui pénètrent dans le substratum, lorsque ce feuillet est appliqué contre la gélose. Dans la nouvelle technique de Spratt et HaAs, qui consiste à cultiver le blastoderme avec son feuillet externe contre l'agar, l'ectoblaste également adhère fortement au substrat. Par conséquent, nous accueillons avec une extrême réserve les affirmations de Spratt et Haas, lesquelles tendent à minimiser l'importance des mouvements morphogénétiques qui ont lieu dans l'ectoblaste.

\section{SUMMARY}

We had already observed that, when the blastoderm is cultured with the endoblast against the agar medium, this layer forms papillae which penetrate in the substrate.

In their new technique, Spratt and HaAs culture the blastoderm with its dorsal face against the substrate, where the endoblast 
as well as the ectoblast is found to be adhering strongly to the agar.

Therefore, we cannot agree with Spratt and Haas, who minimise the importance of the morphogenetic movements in the ectoblast for the primitive streak formation.

\section{ZUSAMMENFASSUNG}

Wir haben früher beobachtet, dass der Endoblast des jungen Keims, auf dem Agar liegend, Papillen bildet, mit welchen er sich an das Substratum festklammert. Mit der neuen Technik von Spratt und HaAs, bei welcher der Keim mit der Rückseite auf das Substratum gelegt wird, verwachst der Ektoblast auch mit dem Agar. So bezweifeln wir sehr die Bestätigungen der beiden Autoren, die die Wichtigkeit der morphogenetischen Bewegungen, welche sich im Ektoblast abspielen, zu sehr unterschätzen.

\section{BIBLIOGRAPHIE}

Gallera, J. et Nicolet, G. 1961. Quelques commentaires sur les méthodes de culture "in vitro" de jeunes blastodermes de Poulet. Experientia. 17: 134.

NEw, D. A. T. 1955. A new technique for cultivation of the chick embryo " in vitro». J. Emb. Exp. Morph. 3: 326-331.

Pasteels, J. 1937. Etudes sur la gastrulation des vertébrés méroblastiques. III Oiseaux. IV Conclusions générales. Arch. Biol. 48: 381-488.

Spratt, N. T. 1947. Development "in vitro" of the early chick blastoderm explanted on yolk and albumen extract saline-Agar substrata. J. Exp. Zool. 106: 345-365.

Spratt, N. T. and HaAs, H. 1960a. Morphogenetic movements in the unincubated chick blastoderm. Anat. Rec. 137: 394.

- 1960. Morphogenetic movements in the lower surface of the unincubated and early chick blastoderm. J. Exp. Zool. 144: 139-158.

- 1960. Importance of morphogenetic movements in the lower surface of the young chick blastoderm. J. Exp. Zool. 144: 257-275.

- 1960. Integrative mechanisms in development of the early chick blastoderm. 1. Regulative potentiality of separated parts. J. Exp. Zool. 145: 97-137. 
Spratt, N. T. and HaAs, H. 1962. Integrative mechanisms in the development of the early chick blastoderm. IV. Synthetic systems composed of parts of different developmental age, synchronisation of developmental rates. J. Exp. Zool. 149: 75-102.

Wetzel, R. 1929. Untersuchungen am Hühnchen. Die Entwicklung des Keimes während der ersten beiden Bruttage. Arch. Entwmech. Org. 110:118-321. 


\section{$2 \mathrm{BHL}$ Biodiversity Heritage Library}

Gallera, Jerzy. 1963. "Culture in vitro des blastodermes de Poulet. Rapports entre les feuillets embryonnaires et le milieu nutritif contenant de la gélose." Revue suisse de zoologie 70, 587-594. https://doi.org/10.5962/bhl.part.75079.

View This Item Online: https://www.biodiversitylibrary.org/item/126487

DOI: https://doi.org/10.5962/bhl.part.75079

Permalink: https://www.biodiversitylibrary.org/partpdf/75079

\section{Holding Institution}

Smithsonian Libraries

\section{Sponsored by}

Biodiversity Heritage Library

\section{Copyright \& Reuse}

Copyright Status: In Copyright. Digitized with the permission of the rights holder

Rights Holder: Muséum d'histoire naturelle - Ville de Genève License: http://creativecommons.org/licenses/by-nc-sa/3.0/ Rights: https://www.biodiversitylibrary.org/permissions/

This document was created from content at the Biodiversity Heritage Library, the world's largest open access digital library for biodiversity literature and archives. Visit BHL at https://www.biodiversitylibrary.org. 